\title{
Controllable Nanostructural Transitions in Grafted Nanoparticle-Block Copolymer Composites
}

\author{
Guang-Kui Xu, Xi-Qiao Feng ( $\varangle)$, and Shou-Wen Yu \\ Institute of Biomechanics and Medical Engineering, Department of Engineering Mechanics, Tsinghua University, Beijing 100084, China \\ Received: 9 February 2010 / Revised: 7 March 2010 / Accepted: 21 March 2010 \\ (C) The Author(s) 2010. This article is published with open access at Springerlink.com
}

\begin{abstract}
We report a theoretical investigation of self-assembled nanostructures of polymer-grafted nanoparticles in a block copolymer and explore underlying physical mechanisms by employing the self-consistent field method. By varying the particle concentration or the chain length and density of the grafted polymer, one can not only create various ordered morphologies (e.g., lamellar or hexagonally packed patterns) but also control the positions of nanoparticles either at the copolymer interfaces or in the center of one-block domains. The nanostructural transitions we here report are mainly attributed to the competition between entropy and enthalpy.
\end{abstract}

\section{KEYWORDS}

Block copolymer, nanocomposite, pattern formation, self-assembly, self-consistent field theory

\section{Introduction}

Dispersing nanoparticles in a block copolymer matrix has emerged as a highly promising way of designing and fabricating novel functional materials for a vast range of applications in, for instance, nanostructured solar cells, photonic band gap materials, highly efficient catalysts, and high-density magnetic storage media [1-5]. The mechanical, optical, and electrical properties of a nanocomposite rely not only on the compositions of its constituent phases but also on its nanostructures and, therefore, achieving high performance nanocomposites often requires precise control of the spatial distribution of the nanoparticles in the polymeric host $[2,5-10]$. To prevent macrophase separation, particles are usually grafted by a brush of short polymers which are chemically identical to one component of the block copolymer. Recent experiments have suggested a facile and effective means of

Address correspondence to fengxq $@$ tsinghua.edu.cn building different self-assembled nanostructures by introducing polymer-grafted nanoparticles in a block copolymer film [6]. Chiu et al. reported that in a poly(styrene- $b$-2-vinylpyridine) (PS- $b$-P2VP) lamellar morphology, thiol-terminated PS-grafted gold nanoparticles are located in PS domains, while particles coated by a mixture of thiol-terminated PS and P2VP reside along the copolymer interfaces [7]. Kim et al. further elaborated this method to determine the areal density of thiol-terminated PS coated on particles above which the particles are located preferentially near the center of PS domains, and below which they clearly segregate to PS/P2VP interfaces [8]. In spite of these encouraging developments, the exact design and fabrication of nanostructures of block copolymerparticle nanocomposites with novel properties and enhanced performance remains a challenging issue.

Various techniques (e.g., the strong segregation method [11], the Monte Carlo method [12, 13], and 
the molecular dynamics method [14]) have been developed to investigate the spatial and temporal evolution of complex polymer systems. Among them, self-consistent field theory (SCFT) has proven to be a powerful method for studying complex morphologies of block copolymers and blends [15-20]. By combining SCFT for inhomogeneous polymers with a density functional theory for hard particles, the self-assembly of bare particles and block copolymers has been studied [21, 22]. A hybrid particle-field method based on SCFT has been developed to investigate the equilibrium structure and properties of mesostructured polymeric fluids with embedded colloids or nanoparticles [20]. More recently, SCFT was further extended to examine the effect of grafted polymer brushes on positioning a single nanoparticle in block copolymers [23]. To date, nevertheless, how to disperse nanoparticles in a desired array in nanocomposites is still an unsolved issue and its solution requires a deep understanding of the physical mechanisms of the formation of various nanostructures. In this paper, therefore, we report a theoretical investigation of self-assembled nanostructures of polymer-grafted nanoparticles in diblock copolymers and explore the underlying physical mechanisms. Especially, the effect of grafted polymer brushes on the nanostructures has been quantitatively examined by a series of SCFT simulations. Moreover, all the interactions between the particles, grafted polymers and copolymer matrix are taken into account in this model. Our numerical experiments demonstrate that by varying the polymergrafted particle content or the density and chain length of grafted polymer, one can not only create different morphologies in nanocomposites, but also design and control the spatial distribution of nanoparticles in order to achieve specific physical properties and functions of materials.

\section{Theoretical model and computational method}

Consider a system containing AB diblock copolymers and polymer-grafted nanoparticles. Each diblock chain consists of $N_{\mathrm{D}}$ segments, while each grafted polymer chain is composed of $\beta N_{D}$ A-type segments. All polymer chains are modeled as flexible Gaussian chains. The volume fraction of A segments per diblock chain is denoted as $f$. For simplicity, assume that $A$ and $B$ segments have the same volume $\rho_{0}^{-1}$ and statistical length $a$. The volume fractions of diblock copolymers and polymer-grafted particles are $\varphi_{\mathrm{D}}$ and $\varphi_{\mathrm{GP}}=1-\varphi_{\mathrm{D}^{\prime}}$ respectively. The particles are approximated as large solvent molecules of radius $R_{\mathrm{p}}$ [24], to which grafted polymer chains are tethered. Only the interaction between $\mathrm{A}$ and $\mathrm{B}$ segments needs to be considered when the grafted polymer chains are long enough to completely shield the particle [25]. However, considering the fact that the grafted polymers are often short in realistic situations, all the interactions $\left(N_{\mathrm{D}} \chi_{\text {IJ }}\right)$ between particles $(\mathrm{P})$, and $\mathrm{A}$ and $\mathrm{B}$ segments are taken into account in this study. In the SCFT method, the pair interactions between different components are determined by a set of effective chemical potential fields, $W_{I}(r)$, denoting the intensity of the mean field felt by the species $I$ at position $r$. The dimensionless free energy of the system is given by

$$
\begin{aligned}
F= & -\varphi_{\mathrm{D}} \ln \left(\frac{Q_{\mathrm{D}}}{V \varphi_{\mathrm{D}}}\right)-\frac{\varphi_{\mathrm{GP}}}{\alpha+\sigma \beta} \ln \left(\frac{Q_{\mathrm{GP}}}{V \varphi_{\mathrm{GP}}}\right) \\
& -\frac{1}{V} \int \mathrm{d} r \sum_{I} W_{I} \phi_{I}+\frac{1}{2 V} \int \mathrm{d} r \sum_{I} \sum_{J \neq I} N_{\mathrm{D}} \chi_{I J} \phi_{I} \phi_{I}
\end{aligned}
$$

where $V$ is the volume of the system, $\sigma$ is the number of grafted polymer chains per particle, $\alpha=4 \pi \rho_{0} R_{\mathrm{P}}^{3} / 3 N_{\mathrm{D}}$ is the volume ratio of a nanoparticle to a diblock chain, and $\phi_{I}(r)$ is the local volume fraction of the species $I$. $Q_{\mathrm{D}}=\int \mathrm{d} r q_{\mathrm{D}}(r, 1)$ and $Q_{\mathrm{GP}}=\int \mathrm{d} \boldsymbol{r} \exp \left[-\alpha W_{\mathrm{P}}(r)\right] q_{\mathrm{G}}(r, 1)^{\sigma}$ are the partition functions of a single diblock chain and a polymer-grafted nanoparticle, respectively.

For a diblock chain, the contour variable $s$ increases continuously from $s=0$ at the free end of A-blocks to $s=1$ at the free end of B-blocks. The spatial coordinate $r$ is normalized by the diblock radius of gyration, $R_{\mathrm{g}}=a \sqrt{N_{\mathrm{D}} / 6}$. The propagator $q_{\mathrm{D}}(r, s)$ represents the probability of finding the $s N_{D}$-th segment at position $r$, which satisfies the modified diffusion equation

$$
\frac{\partial q_{\mathrm{D}}(r, s)}{\partial s}=\nabla^{2} q_{\mathrm{D}}(r, s)-W_{I}(r) q_{\mathrm{D}}(r, s)
$$

subject to the initial condition $q_{\mathrm{D}}(r, 0)=1$. The field 
$W_{I}(r)$ switches from $W_{\mathrm{A}}(r)$ for $0<s<f$ to $W_{\mathrm{B}}(r)$ for $s>f$. The grafted polymer chain propagator $q_{\mathrm{G}}(r, s)$ satisfies [26]

$$
\frac{\partial q_{\mathrm{G}}(r, s)}{\partial s}=\beta \nabla^{2} q_{\mathrm{G}}(r, s)-\beta W_{\mathrm{A}}(r) q_{\mathrm{G}}(r, s)
$$

with the initial condition $q_{\mathrm{G}}(r, 0)=1$ for the free end of the chain at $s=0$. The complementary propagators $q_{\mathrm{D}}^{+}(\boldsymbol{r}, s)$ and $q_{\mathrm{G}}^{+}(\boldsymbol{r}, s)$ respectively obey equations similar to Eqs. (2) and (3) except that their right-hand sides are multiplied by -1 . The corresponding initial conditions are $q_{\mathrm{D}}^{+}(r, 1)=1$ and $q_{\mathrm{G}}^{+}(r, 1)=\exp \left[-\alpha W_{\mathrm{P}}(r)\right] q_{\mathrm{G}}(r, 1)^{\sigma-1}$, respectively. In terms of these propagators, the local densities of different components are calculated by

$$
\begin{aligned}
\phi_{\mathrm{A}}(\boldsymbol{r})= & \frac{\varphi_{\mathrm{D}} V}{Q_{\mathrm{D}}} \int_{0}^{f} \mathrm{~d} s q_{\mathrm{D}}(\boldsymbol{r}, s) q_{\mathrm{D}}^{+}(\boldsymbol{r}, s) \\
& +\frac{\varphi_{\mathrm{GP}} \sigma \beta V}{(\alpha+\sigma \beta) Q_{\mathrm{GP}}} \int_{0}^{1} \mathrm{~d} s q_{\mathrm{G}}(\boldsymbol{r}, s) q_{\mathrm{G}}^{+}(\boldsymbol{r}, s) \\
\phi_{\mathrm{B}}(\boldsymbol{r})= & \frac{\varphi_{\mathrm{D}} V}{Q_{\mathrm{D}}} \int_{f}^{1} \mathrm{~d} s q_{\mathrm{D}}(\boldsymbol{r}, s) q_{\mathrm{D}}^{+}(\boldsymbol{r}, s) \\
\phi_{\mathrm{P}}(\boldsymbol{r})= & \frac{\varphi_{\mathrm{GP}} \alpha V}{(\alpha+\sigma \beta) Q_{\mathrm{GP}}} \exp \left[-\alpha W_{\mathrm{P}}(\boldsymbol{r})\right] q_{\mathrm{G}}(\boldsymbol{r}, 1)^{\sigma}
\end{aligned}
$$

By minimizing the free energy $F$ with respect to $\phi_{I}(r)$, one has

$$
W_{I}(\boldsymbol{r})=\sum_{I \neq J} N_{\mathrm{D}} \chi_{I J} \phi_{J}(\boldsymbol{r})+\xi(r)
$$

where $\xi$ is a Lagrange multiplier. Equations (4)-(7), in conjunction with the incompressible condition $\sum_{I} \phi_{I}(r)=1$, form a closed set of equations that can be solved self-consistently in real space. To obtain the equilibrium structure, we solve these equations by using the combinatorial screening technique of Drolet and Fredrickson [16] implemented with a highly stable and accurate numerical algorithm [27, 28]. All the simulations are performed in a two-dimensional $128 \times 128$ square lattice with periodic boundary conditions and the grid size $\Delta x=0.1$.

\section{Results and discussion}

To verify the efficacy of the calculation method and compare with the experiments of PS-grafted Au particles in a PS- $b$-P2VP copolymer $[6,8,20]$, we regard PS and P2VP chains as A- and B-blocks, respectively. To mimic the fact that $\mathrm{Au}$ particles are selective to B-blocks [8], we take the interaction values of $\mathrm{Au}$ particles with A- and B-blocks as $N_{\mathrm{D}} \chi_{\mathrm{AP}}=16$ and $N_{\mathrm{D}} \chi_{\mathrm{BP}}=2$, respectively. The other parameter values are used: $N_{\mathrm{D}} \chi_{\mathrm{AB}}=16$ and $\alpha=0.1$.

We first examine the transition of self-assembled nanostructures with respect to the increase in the A-grafted particle filling fraction, $\varphi_{\mathrm{GP}}$, which has proven to be a key factor in producing different structures in a block copolymer film [6]. In a symmetric diblock copolymer $(f=0.5)$, the blend self-assembles into a lamellar morphology at a low particle content (e.g., $\varphi_{\mathrm{GP}}=0.15$ ), as shown in Fig. 1(a), where the nanoparticles form a thin lamella located in the center of A-domains. As $\varphi_{\mathrm{GP}}$ increases, B segments (red) and particles (yellow) respectively form hexagonally packed cylinders and dumbbells, both of which are embedded into the A-block matrix (blue), as shown in Fig. 1(b). This morphology transition can be understood as follows. On the one hand, additional nanoparticles swell and, in turn, distort the A lamellar domains to gain more translational entropy, and, on the other hand, the reduced $B$ segments aggregate into cylinders, rendering a smaller contact area with the A phase and thus a reduction of enthalpic energy. This result is consistent with recent experimental observations $[6,20]$. In the case of an asymmetric diblock copolymer (e.g., $f=0.3$ ), a regular hexagonal pattern forms in the blend with a low particle filling fraction (e.g., $\varphi_{\mathrm{GP}}=0.05$ ), where the particles are located in the circular A-domains, as shown in Fig. 1(c). A transition from hexagonal to lamellar morphology occurs when a critical particle concentration is reached (Fig. 1(d)), because the addition of grafted polymer chains acts to increase the effective volume fraction of A segments in the system. This concurs with the experimental and numerical results reported by Side et al. [20]. Our simulations demonstrate that for various diblock compositions, one can obtain different ordered nanostructures by adjusting the polymer-grafted particle concentration.

As is well known, the spatial distribution of nanoparticles may have an important or even dominant influence on some properties and functions of 


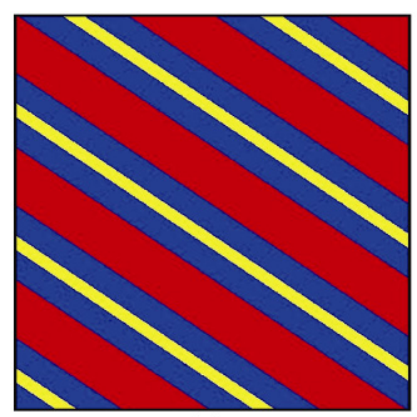

(a)

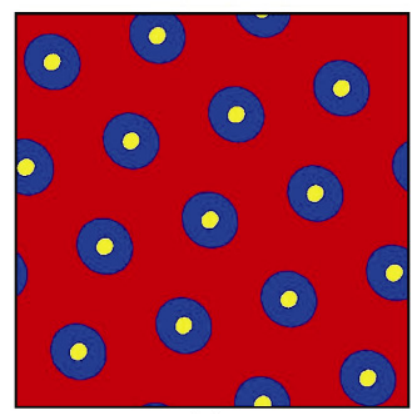

(c)

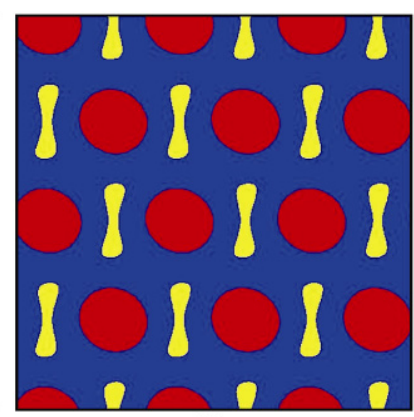

(b)

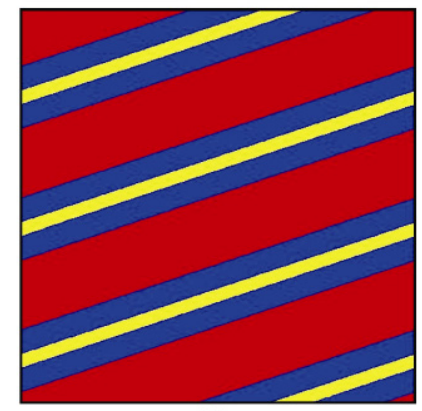

(d)
Figure 1 Aggregate morphologies of diblock copolymer and polymer-grafted nanoparticle blends for $\sigma=6$ and $\beta=0.05$. For the symmetric diblock copolymer, we take (a) $\varphi_{\mathrm{GP}}=0.15$ and (b) $\varphi_{\mathrm{GP}}=0.4$. For the asymmetric diblock copolymer $(f=0.3)$, we assume (c) $\varphi_{\mathrm{GP}}=0.05$ and (d) $\varphi_{\mathrm{GP}}=0.3$. The blue, red, and yellow colors represent A-blocks, B-blocks, and particles, respectively

nanocomposites. In what follows, our attention will be focused on the precise control of particle positions in, for instance, a symmetric diblock-copolymer lamellar system. As pointed out by Akcora et al. [29], the grafting chain density and the grafted chain length are two key and easily controlled factors in determining the self-assembled structures, which will be quantitatively examined in the sequel. In our calculations, the concentration of A-grafted particles is fixed at $\varphi_{\mathrm{GP}}=0.15$ to match with the experiments of $\mathrm{Kim}$ et al. [8]. For three representative grafting chain densities $(\sigma)$, Fig. 2 illustrates the corresponding spatial distributions of the grafted nanoparticles. At a low chain density (e.g., $\sigma=5$ ), particles preferentially segregate to the A/B interfaces, as shown in Fig. 2(a). As $\sigma$ increases (e.g., $\sigma=9$ ), the preferable interaction between particles and B segments is partly shielded by the grafted A-chains. Therefore, some particles locate along the $\mathrm{A} / \mathrm{B}$ interfaces while the others are embedded near the center of A-domains (Fig. 2(b)). For a very large value of $\sigma$, the particles are fully shielded by the grafted A-chains, resulting in the concentration of all particles in A-domains, as illustrated in Fig. 2(c), where $\sigma=13$. For greater clarity, the precise particle positions are illustrated schematically in the insets of the figures. This transition of particle positions with the increase of the grafting chain density is in agreement with relevant experimental observations [8]. When the grafting density is low, the B segments can interpenetrate into the grafted A-brushes and interact with the particles directly. For a higher grafting chain density, B segments cannot easily penetrate into the grafted A-brushes, resulting in a loss in conformational entropy of the B-blocks. Simultaneously, the particles segregate to the center of A-domains, as a requirement of the minimization of the contact area between grafted A-brushes and B-blocks and a reduction in enthalpy. In this case, therefore, enthalpy plays a more predominant role than entropy in determining the spatial distribution of the nanoparticles.

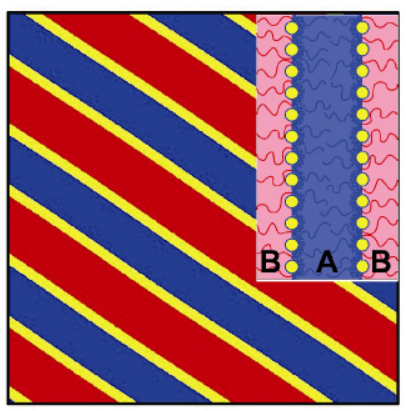

(a)

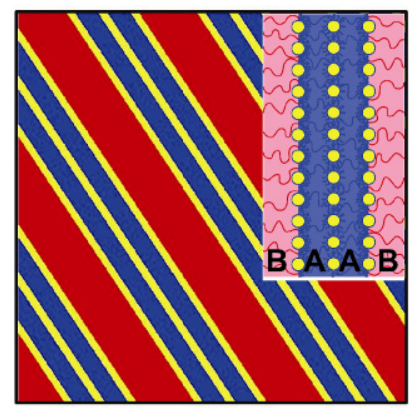

(b)

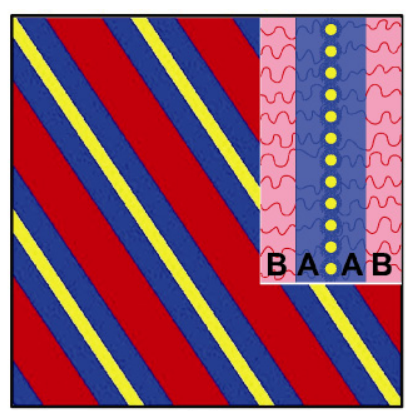

(c)

Figure 2 Influence of the number $\sigma$ of grafted chains per particle on the particle distribution for $\beta=0.025$ : (a) $\sigma=5$, (b) $\sigma=9$, and (c) $\sigma=13$. A larger value of $\sigma$ indicates a higher grafting chain density. The insets in the figures show particle positions. The blue, red, and yellow colors represent A-blocks, B-blocks, and particles, respectively 
Furthermore, as the grafted chain length increases, a transition in the spatial distribution of nanoparticles (figures not shown), which is quite similar to that induced by increasing the grafting chain density, is observed in our simulations. It is easy to understand that both the chain length and density of grafted polymers play a shielding role with respect to the particle-B segment interaction. Long grafted polymer chains can fully shield the particles and drive them to the center of A-domains due to the repulsion between the grafted A-chains and B-blocks. To more clearly show the dependence of particle locations on the grafted chain length and grafting chain density, a phase diagram for the self-assembled lamellar structures is given in Fig. 3 on the basis of a large number of simulations. The diagram is divided into three regions, $\mathrm{I},(\mathrm{I}+\mathrm{C})$, and $\mathrm{C}$, where I corresponds to the case where all particles segregate along copolymer interfaces and $C$ to the case where all particles are embedded in the center of A-domains. The particle position transitions in the order of $\mathrm{I} \rightarrow(\mathrm{I}+\mathrm{C}) \rightarrow \mathrm{C}$ can be achieved by increasing either the grafting chain density or the grafted chain length. Such a phase diagram allows us to predict the particle position in a block copolymer template under specified conditions, which is of importance for engineering nanocomposites with enhanced properties.

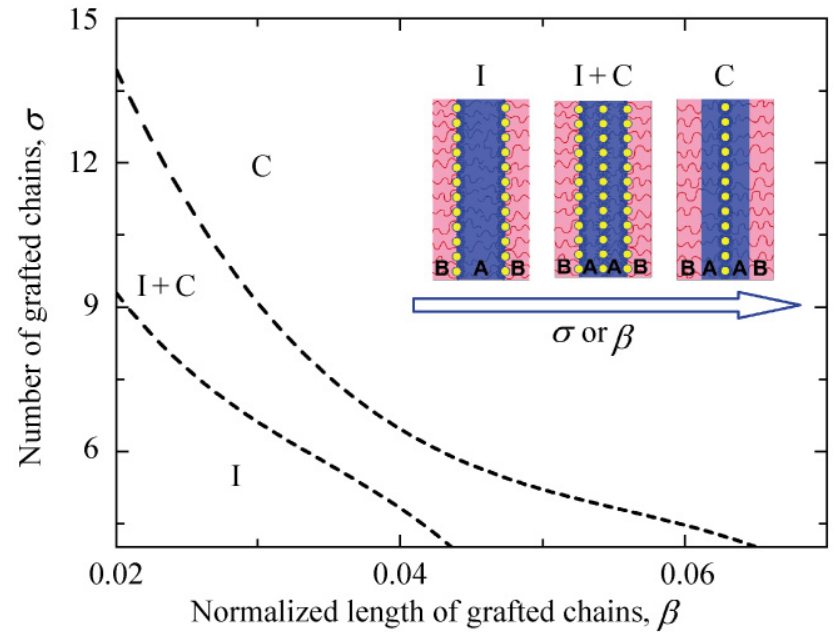

Figure 3 A phase diagram illustrating the dependence of particle location on the number $\sigma$ and normalized length $\beta$ of grafted chains. The notations I and $\mathrm{C}$ stand for particles locating along the copolymer interfaces and the center of A-domains, respectively. The inset shows the nanostructural transition with increasing $\sigma$ or $\beta$
To quantitatively explore the physical mechanisms underlying the formation of the above-described nanostructures, we calculate the entropic and enthalpic contributions to the total free energy, as shown in Fig. 4. In Eq. (1), the first three terms on the right-hand side represent the contribution of entropy to the free energy of system while the last term gives the enthalpic energy. It can be seen from Fig. 4 that the entropic contribution to free energy, $F_{\mathrm{S}}$, increases with the increase in the grafting chain density $(\sigma)$ or the grafted chain length $(\beta)$. This is because B-blocks cannot penetrate into the denser grafted A-brushes for large

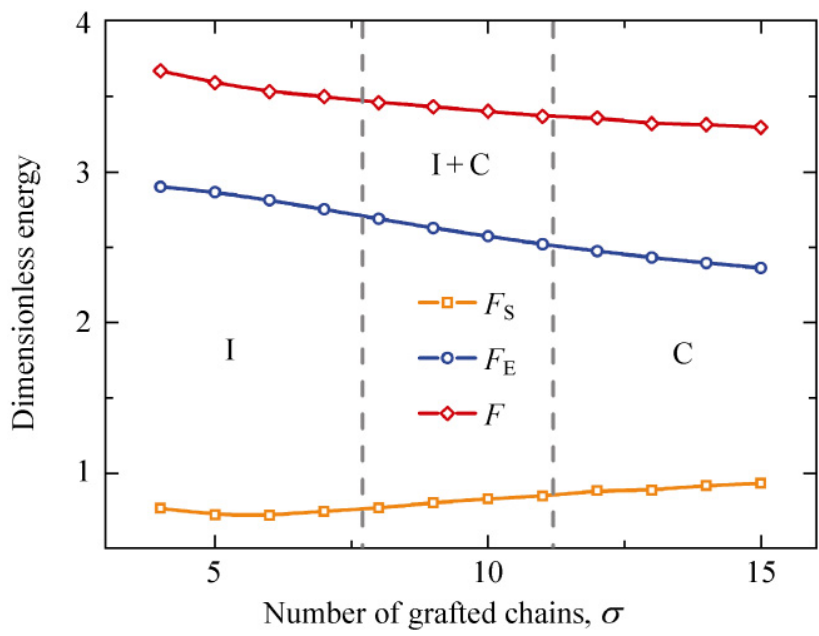

(a)

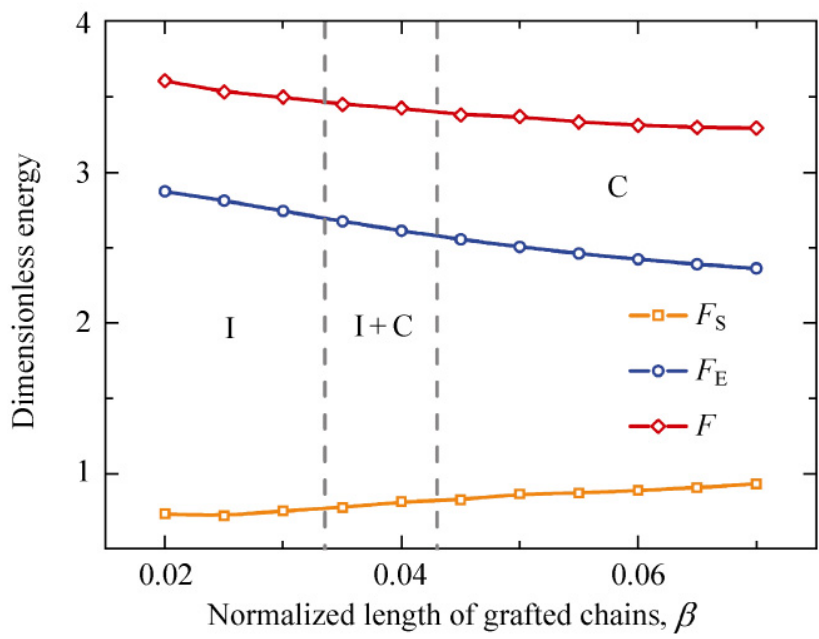

(b)

Figure 4 Contribution of entropy $\left(F_{\mathrm{S}}\right)$ and enthalpy $\left(F_{\mathrm{E}}\right)$ to the total free energy $F$ as functions of (a) the number $\sigma$ and (b) the normalized length $\beta$ of grafted polymer chains. The dashed lines represent the transition boundaries. We take $\beta=0.025$ in (a) and $\sigma=6$ in (b) 
values of $\sigma$ or $\beta$. The dense grafted brushes confine the degrees of freedom of B-blocks and cause a loss in the conformational entropy of the copolymer. Otherwise, the grafted nanoparticles will migrate into the energetically favorable A-domains and lead to a decrease in the enthalpic free energy $F_{\mathrm{E}}$ as a result of the repulsion between the dense grafted brushes and B-segments. Therefore, the final particle distribution within self-assembled nanostructures relies on the competition between entropic and enthalpic energies. A delicate balance of entropy and enthalpy can be utilized to elaborately tailor the particle distribution in the nanostructures, which holds great promise in the area of fabricating novel functional nanomaterials.

\section{Conclusions}

In summary, how to control the self-assembled nanostructures of block copolymer-nanoparticle blends has been explored with the aim of engineering nanocomposites that exhibit advantageous optical, electrical, or mechanical properties. This study suggests that the structural morphology of polymernanoparticle composites is tunable by functionalizing or grafting the added nanoparticles. The particles can further be positioned either in a specific polymer phase or along the interfaces between two different phases by proper selection of the concentration, length, and density of the grafted polymer chains. The observed nanostructural transitions are attributed to the competition between entropy and enthalpy. Finally, it is emphasized that more delicate nanostructures can be achieved by further utilization of some other factors (e.g., size and shape of particles $[5,30])$, which can also be studied by the SCFT method used in this work. Future work will include exploiting the effect of grafted nanoparticles in the formation of hierarchical structures, which opens a fascinating way to design and synthesize bioinspired composites with superior physical properties and multiple functions.

\section{Acknowledgements}

Support from the National Natural Science Foundation of China (Nos. 10972121, 10732050, and 10772093), the
Ministry of Education (SRFDP 20090002110047), and the 973 program of MOST (No. 2010CB631005) are acknowledged.

Open Access: This article is distributed under the terms of the Creative Commons Attribution Noncommercial License which permits any noncommercial use, distribution, and reproduction in any medium, provided the original author(s) and source are credited.

\section{References}

[1] Balazs, A. C.; Emrick, T.; Russell, T. P. Nanoparticle polymer composites: where two small worlds meet. Science 2006, 314, 1107-1110.

[2] Bockstaller, M. R.; Thomas, E. L. Proximity effects in selforganized binary particle-block copolymer blends. Phys. Rev. Lett. 2004, 93, 166106.

[3] Liff, S. M.; Kumar, N.; McKinley, G. H. High-performance elastomeric nanocomposites via solvent-exchange processing. Nat. Mater. 2007, 6, 76-83.

[4] Warren, S. C. ; Messina, L. C.; Slaughter, L. S.; Kamperman, M.; Zhou, Q.; Gruner, S. M.; DiSalvo, F. J.; Wiesner, U. Ordered mesoporous materials from metal nanoparticle-block copolymer self-assembly. Science 2008, 320, 1748-1752.

[5] Bockstaller, M. R.; Mickiewicz, R. A.; Thomas, E. L. Block copolymer nanocomposites: perspectives for tailored functional materials. Adv. Mater. 2005, 17, 1331-1349.

[6] Kim, B. J.; Chiu, J. J.; Yi, G. R.; Pine, D. J.; Kramer, E. J. Nanoparticle-induced phase transitions in diblock-copolymer films. Adv. Mater. 2005, 17, 2618-2622.

[7] Chiu, J. J.; Kim, B. J.; Kramer, E. J.; Pine, D. J. Control of nanoparticle location in block copolymers. J. Am. Chem. Soc. 2005, 127, 5036-5037.

[8] Kim, B. J.; Bang, J.; Hawker, C. J.; Kramer, E. J. Effect of areal chain density on the location of polymer-modified gold nanoparticles in a block copolymer template. Macromolecules 2006, 39, 4108-4114.

[9] Kalra, V.; Lee, J.; Lee, J. H.; Lee, S. G.; Marquez, M.; Wiesner, U.; Joo, Y. L. Controlling nanoparticle location via confined assembly in electrospun block copolymer nanofibers. Small 2008, 4, 2067-2073.

[10] Fu, S. Y.; Feng, X. Q.; Lauke, B; Mai, Y. W. Effects of particle size, particle/matrix interface adhesion and particle loading on mechanical properties of particulate polymer composites. Compos. Part B: Eng. 2008, 39, 933-961.

[11] Pryamitsyn, V.; Ganesan, V. Strong segregation theory of block copolymer-nanoparticle composites. Macromolecules 2006, 39, 8499-8510. 
[12] Wang, Q.; Nealey, P. F.; de Pablo, J. J. Behavior of single nanoparticle/homopolymer chain in ordered structures of diblock copolymers. J. Chem. Phys. 2003, 118, 1127811285.

[13] Huh, J.; Ginzburg, V. V.; Balazs, A. C. Thermodynamic behavior of particle/diblock copolymer mixtures: Simulation and theory. Macromolecules 2000, 33, 8085-8096.

[14] Schultz, A. J.; Hall, C. K.; Genzer, J. Computer simulation of block copolymer/nanoparticle composites. Macromolecules 2005, 38, 3007-3016.

[15] Matsen, M. W. The standard Gaussian model for block copolymer melts. J. Phys.: Condens. Matter 2002, 14, R21R47.

[16] Drolet, F.; Fredrickson, G. H. Combinatorial screening of complex block copolymer assembly with self-consistent field theory. Phys. Rev. Lett. 1999, 83, 4317-4320.

[17] Guo, Z. J.; Zhang, G. J.; Qiu, F.; Zhang, H. D.; Yang, Y. L.; Shi, A. C. Discovering ordered phases of block copolymers: New results from a generic Fourier-space approach. Phys. Rev. Lett. 2008, 101, 028301.

[18] Xu, G. K.; Li, Y.; Li, B.; Feng, X. Q.; Gao, H. J. Self-assembled lipid nanostructures encapsulating nanoparticles in aqueous solution. Soft Matter 2009, 5, 3977-3983.

[19] Xu, G. K.; Feng, X. Q.; Li, Y. Self-assembled nanostructures of homopolymer and diblock copolymer blends in a selective solvent. J. Phys. Chem. B 2010, 114, 1257-1263.

[20] Sides, S. W.; Kim, B. J.; Kramer, E. J.; Fredrickson, G. H. Hybrid particle-field simulations of polymer nanocomposites. Phys. Rev. Lett. 2006, 96, 250601.

[21] Thompson, R. B.; Ginzburg, V. V.; Matsen, M. W.; Balazs, A. C. Predicting the mesophases of copolymer-nanoparticle composites. Science 2001, 292, 2469-2472.
[22] Lee, J. Y.; Shou, Z. Y.; Balazs, A. C. Modeling the selfassembly of copolymer-nanoparticle mixtures confined between solid surfaces. Phys. Rev. Lett. 2003, 91, 136103.

[23] Kim, J. U.; Matsen, M. W. Positioning Janus nanoparticles in block copolymer scaffolds. Phys. Rev. Lett. 2009, 102, 078303.

[24] Spontak, R. J.; Shankar, R.; Bowman, M. K.; Krishnan, A. S.; Hamersky, M. W.; Samseth, J.; Bockstaller, M. R.; Rasmussen, K. Ø. Selectivity- and size-induced segregation of molecular and nanoscale species in microphase-ordered triblock copolymers. Nano Lett. 2006, 6, 2115-2120.

[25] Reister, E.; Fredrickson, G. H. Phase behavior of a blend of polymer-tethered nanoparticles with diblock copolymers. $J$. Chem. Phys. 2005, 123, 214903.

[26] Patel, D. M.; Fredrickson, G. H. Quenched and annealed disorder in randomly grafted copolymer melts. Phys. Rev. E 2003, 68, 051802.

[27] Tzeremes, G.; Rasmussen, K. Ø.; Lookman, T.; Saxena, A. Efficient computation of the structural phase behavior of block copolymers. Phys. Rev. E 2002, 65, 041806.

[28] Sides, S. W.; Fredrickson, G. H. Parallel algorithm for numerical self-consistent field theory simulations of block copolymer structure. Polymer 2003, 44, 5859-5866.

[29] Akcora, P.; Liu, H. J.; Kumar, S. K.; Moll, J.; Li, Y.; Benicewicz, B. C.; Schadler, L. S.; Acehan, D.; Panagiotopoulos, A. Z.; Pryamitsyn, V.; Ganesan, V.; Ilavsky, J.; Thiyagarajan, P.; Colby, R. H.; Douglas, J. F. Anisotropic self-assembly of spherical polymer-grafted nanoparticles. Nat. Mater. 2009, 8, 354-359.

[30] Kim, J. U.; O'Shaughnessy, B. Morphology selection of nanoparticle dispersions by polymer media. Phys. Rev. Lett. 2002, 89, 238301. 